\title{
Do people with social anxiety feel anxious about interacting with a robot?
}

\author{
Tatsuya Nomura ${ }^{1,3} \cdot$ Takayuki Kanda $^{2,3} \cdot$ Tomohiro Suzuki $^{4} \cdot$ Sachie Yamada ${ }^{5}$
}

Received: 21 April 2018 / Accepted: 23 March 2019 / Published online: 9 April 2019

(c) The Author(s) 2019

\begin{abstract}
To investigate whether people with social anxiety have less actual and "anticipatory" anxiety when interacting with a robot compared to interacting with a person, we conducted a $2 \times 2$ psychological experiment with two factors: social anxiety and interaction partner (a human confederate and a robot). The experiment was conducted in a counseling setting where a participant played the role of a client and the robot or the confederate played the role of a counselor. First, we measured the participants' social anxiety using the Social Avoidance and Distress Scale, after which, we measured their anxiety at two specific moments: "anticipatory anxiety" was measured after they knew that they would be interacting with a robot or a human confederate, and actual anxiety was measured after they actually interacted with the robot or confederate. Measurements were performed using the Profile of Mood States and the State-Trait Anxiety Inventory. The results indicated that participants with higher social anxiety tended to feel less "anticipatory anxiety" and tension when they knew that they would be interacting with robots compared with humans. Moreover, we found that interaction with a robot elicited less tension compared with interaction with a person regardless of the level of social anxiety.
\end{abstract}

Keywords Communication robots $\cdot$ Social anxiety $\cdot$ Anticipatory anxiety

\section{Introduction}

Tatsuya Nomura

nomura@ rins.ryukoku.ac.jp

Takayuki Kanda

kanda@i.kyoto-u.ac.jp

Tomohiro Suzuki

suzukirt_@nifty.com

Sachie Yamada

s-yamada@tokai-u.jp

1 Department of Media Informatics, Ryukoku University, 1-5 Yokotani, Seta-Ohe-Cho, Otsu, Shiga 520-2194, Japan

2 Department of Informatics, Kyoto University, Yoshida-honmachi, Kyoto 606-8501, Japan

3 ATR Intelligent Robotics and Communication Laboratories, 2-2 Hikaridai, Seika-cho, Soraku-gun, Kyoto 619-0288, Japan

4 School of Child Psychology, Tokyo Future University, 34-12 Senju-akebono, Adachi-ku, Tokyo 120-0023, Japan

5 Department of Psychological and Sociological Studies, Tokai University, 4-1-1 Kitakaname, Hiratsuka, Kanagawa 259-1292, Japan
People with social anxiety feel nervous in social situations, such as when they talk with other people, meet someone for the first time, or give a speech. Such individuals often fail to communicate with others appropriately, and thereby fail to perform social tasks reasonably. People who experience excessive amounts of social anxiety are often diagnosed as having social anxiety disorder; these individuals have more difficulty performing activities of daily living, such as studying at school and working in an office (American Psychiatric Association 2013). The lifetime prevalence of social anxiety disorder has been reported to be about $13 \%$, and about $20 \%$ of the population are thought to have strong fear when speaking or performing tasks in front of others (American Psychiatric Association 1994). Therefore, the development of training programs that help such individuals moderate their social anxiety is needed (Schneier et al. 1992).

People with social anxiety start to feel anxious when they simply anticipate communicating with others, even before any communication actually takes place; this type of anxiety is referred to as "anticipatory anxiety". People with anticipatory anxiety tend to avoid taking advantage of 
various useful opportunities. For instance, if such an individual were about to go to work, he/she would feel anxious in anticipation of receiving negative evaluations from other commuters, and in serious cases, even avoid going out at all. Likewise, these individuals feel anxious when they are about to go shopping or meet friends. Their anticipatory anxiety is evoked whenever communication with someone is expected, and sometimes causes them to avoid receiving appropriate services. Nevertheless, many services are provided via communication, e.g., education and consultation. One notable setting can be a counseling setting. When a person has a problem, one of the most important ways to cope with it is to consult with experts; in such a situation, these individuals are required to talk about themselves. However, this situation often involves meeting a stranger and opening up to them. Individuals with a high level of social anxiety feel anxious in this type of situation, and as a result, show a tendency toward avoidance. In fact, it has been reported that only about half of those with social anxiety disorder receive proper treatment, with initial treatment not typically received until after experiencing symptoms for 15-20 years (American Psychiatric Association 2013). One must decide to meet with a counselor, and while this may be a trivial decision for many, it is not easy for those with social anxiety disorder. Counseling is normally provided via face-to-face communication, so it causes people with social anxiety disorder to feel anxious; because of this "anticipatory anxiety", such individuals tend to avoid counseling altogether.

Given this background, we decided to investigate whether a robot could be a useful solution for people with social anxiety. For instance, if they know that they will be meeting a robot for counseling, will they feel a level of anxiety similar to that they feel for humans? We hypothesized that such people would feel less anxious when meeting a robot counselor. The literature suggests that persons with social anxiety tend to prefer computer-mediated communication with others and interaction with artifacts such as robots over face-to-face communication with others (Kang and Gratch 2010). Moreover, persons with a strong fear of negative evaluations, which is a core symptom of social anxiety, tend to evaluate interactions with robots positively (Nomura and Kanda 2015). A previous survey-based study reported that people with social anxiety prefer interaction with robots over humans in many situations (Suzuki et al. 2015).

However, to our knowledge, no experimental study has investigated the influence of social anxiety on human-robot interactions. Although a fear of negative evaluations has been suggested in cases in which humans are evaluated by robots (Nomura and Kanda 2015), whether people with social anxiety prefer robots or humans as communication partners remains unclear. If people with social anxiety find it easier to communicate with robots than with humans under social situations in which they are exposed to substantial burdens, interaction with communication robots could be expected to be helpful.

Considering the above situation, we investigated the following hypotheses:

- Persons with social anxiety would have less "anticipatory anxiety" when they anticipate meeting with a robot than when they anticipate meeting with a person

- Persons with social anxiety would have less (actual) anxiety after interacting with a robot than after interacting with a person

To investigate the above hypotheses, we conducted a psychological experiment based on a comparison of interactions with a robot and a human. Herein, we report the experimental results and discuss their implications.

\section{Methods}

The experiment in the present study was conducted in a setting where a robot/human confederate served as a counselor who exhibited listening behaviors. The study participants were then asked to talk to the counselor. We recruited participants via a website for recruiting part-time workers. The study participants were 19 Japanese university students (males: 11, females: 8) with a mean age of 21.3 years (standard deviation $[\mathrm{SD}]=1.9$ years). The participants were paid for their participation. This study was approved by the ethical review board at ATR Intelligent Robotics and Communication Laboratories.

\subsection{Robot used in the experiment}

We used the Robovie communication robot (Ishiguro et al. 2001). As shown in Fig. 1, Robovie has a humanlike appearance and was designed for communication with humans. It stands $120 \mathrm{~cm}$ tall with a diameter of $40 \mathrm{~cm}$ and weighs about $40 \mathrm{~kg}$. The robot has two arms $(4 \times 2 \mathrm{DOF})$, a head ( $3 \mathrm{DOF})$, and two eyes $(2 \times 2 \mathrm{DOF}$ for gaze control). Although the robot can move around using two wheels at the bottom of its body, this function was not used in the present experiment.

The participants were instructed that the robot could understand humans' utterances and speak although the robot was actually controlled by Wizard-of-Oz method.

\subsection{Measures}

We used the following scales for the measurements: 


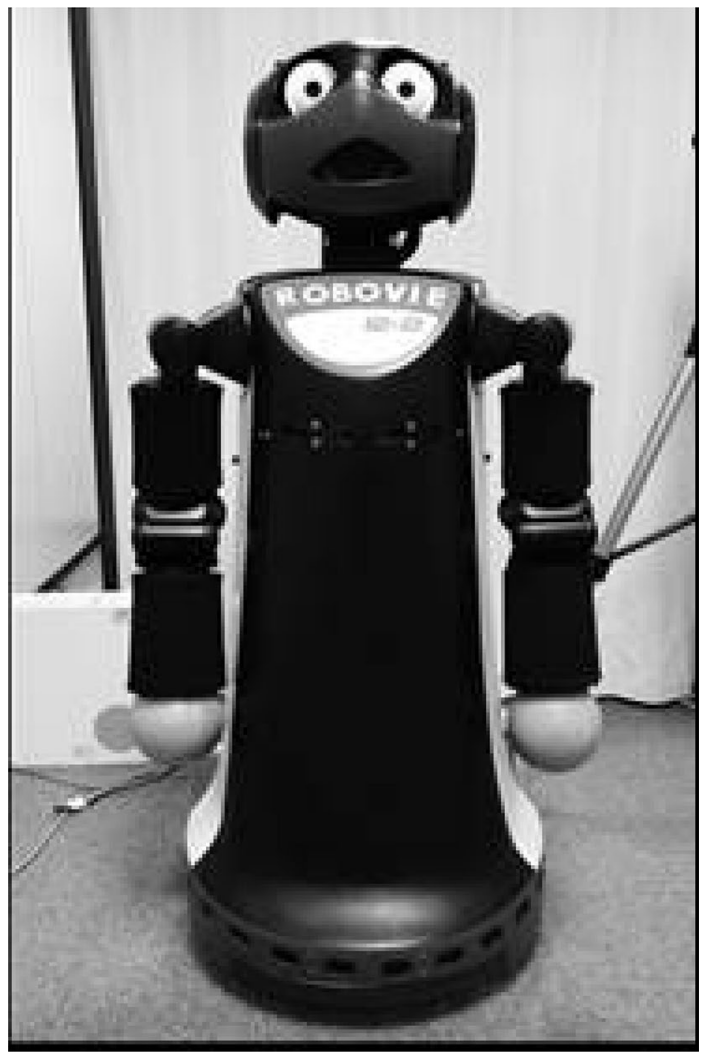

Fig. 1 The "Robovie" robot used in the experiment

\subsubsection{Social anxiety trait}

We aimed to measure the participants' social anxiety trait (in psychology, a trait is considered a stable characteristic of an individual that is sustained across different situations). For this purpose, we used the Japanese version of the Social Avoidance and Distress Scale (SADS) (Watson and Friend 1969). The SADS is composed of 28 items showing individual tendencies of avoidance and distress in social situations (e.g., "I feel tension and nervous when I am introduced to others."). Each item has a rating score of "yes $(=1)$ " or "no $(=0)$ ".

\subsubsection{State anxiety}

At a couple of moments during the experiment, we aimed to measure the degree to which each participant felt anxious. Therefore, we used the Japanese version of the State-Trait Anxiety Inventory-State Version (STAI-S) for state anxiety (in psychology, a mental/psychological state is considered to change depending on different situations) (Spielberger et al. 1983). Twenty items were adopted to measure state anxiety (e.g., "I feel anxious", "I feel apprehensive"). Each item is rated on a four-point Likert-type scale (from 1 "not at all" to 4 "extremely") in regard to how strongly she/he feels about a situation.

\subsubsection{Tension}

At a couple of moments during the experiment, we measured the participants' tension using the Japanese version of the Profile of Mood States (POMS) (McNair et al. 1971). The POMS is a psychological scale that can measure temporal moods and affective states in humans; these changes depending on the situation. Among six subscales of the POMS, we used the tension-anxiety subscale (five items, including "anxious" and "nervous"). Each item is rated on a five-point Likert-type scale (from 0 "not at all" to 4 "extremely") in regard to how strongly she/he feels about a situation.

\subsection{Conditions}

The present experiment used a $2 \times 2$ mixed design with the following experimental conditions:

- Interaction partner

- Robot: Participants interacted with a robot, "Robovie", as explained in Sect. 2.1.

- Human: participants interacted with a human confederate. There were two confederates, one male and one female. To control the confederates' reactions to the participants, they were trained in advance to ensure that they would exhibit similar listening behaviors (the details of these behaviors are explained below). Each confederate was assigned to interact with a participant of the opposite gender.

The interaction partner was the within-participant factor. The order of interaction with the robot and human was counter-balanced.

\section{- Social anxiety trait of the participants}

This was the between-participant factor. The participants were divided into two groups based on their social anxiety trait (as measured by the SADS) scores as follows:

- "lower" social anxiety: participants whose social anxiety trait (SADS) score was lower than the median for all participants were categorized as having lower social anxiety.

- "higher" social anxiety: participants whose social anxiety trait (SADS) score was higher than the median of all participants were categorized as having higher social anxiety. 


\subsection{Procedure}

The basic experimental procedure was conducted as follows (Fig. 2).

\section{Step 1 Baseline measurement}

Each participant received a brief explanation about the experiment and signed a consent form. The experimenter informed the participant that he/she would play the role of the client and the robot or the human would play the role of the counselor. Next, we had the participant wait for $10 \mathrm{~min}$ so that they would be relaxed.

We then conducted the baseline measurements. We administrated the questionnaire explained in the measurement section. That is, we measured the participant's social anxiety trait using the SADS; tension and state anxiety were measured using the POMS and STAI-S, respectively, as baseline values when the participant was relaxed. The participant was then asked to wait for another $5 \mathrm{~min}$.

Step 2 Measurement of anticipatory anxiety

Next, we measured the participants' anticipatory anxiety. Here, the experimenter instructed the participant that she/he would talk to a robot or a human counselor about contents related to herself/himself for $5 \mathrm{~min}$ in an experimental room (Fig. 3). This meant that the participant would now anticipate interaction with the robot or the human. Immediately after issuing these instructions, we administrated the questionnaire to measure their tension and state anxiety using the POMS and STAI-S.

Step 3 Interaction with a robot/person

Next, we told the participants that a human/robot communication partner (counselor) was in a room, and that he/
Fig. 2 Basic experimental procedure

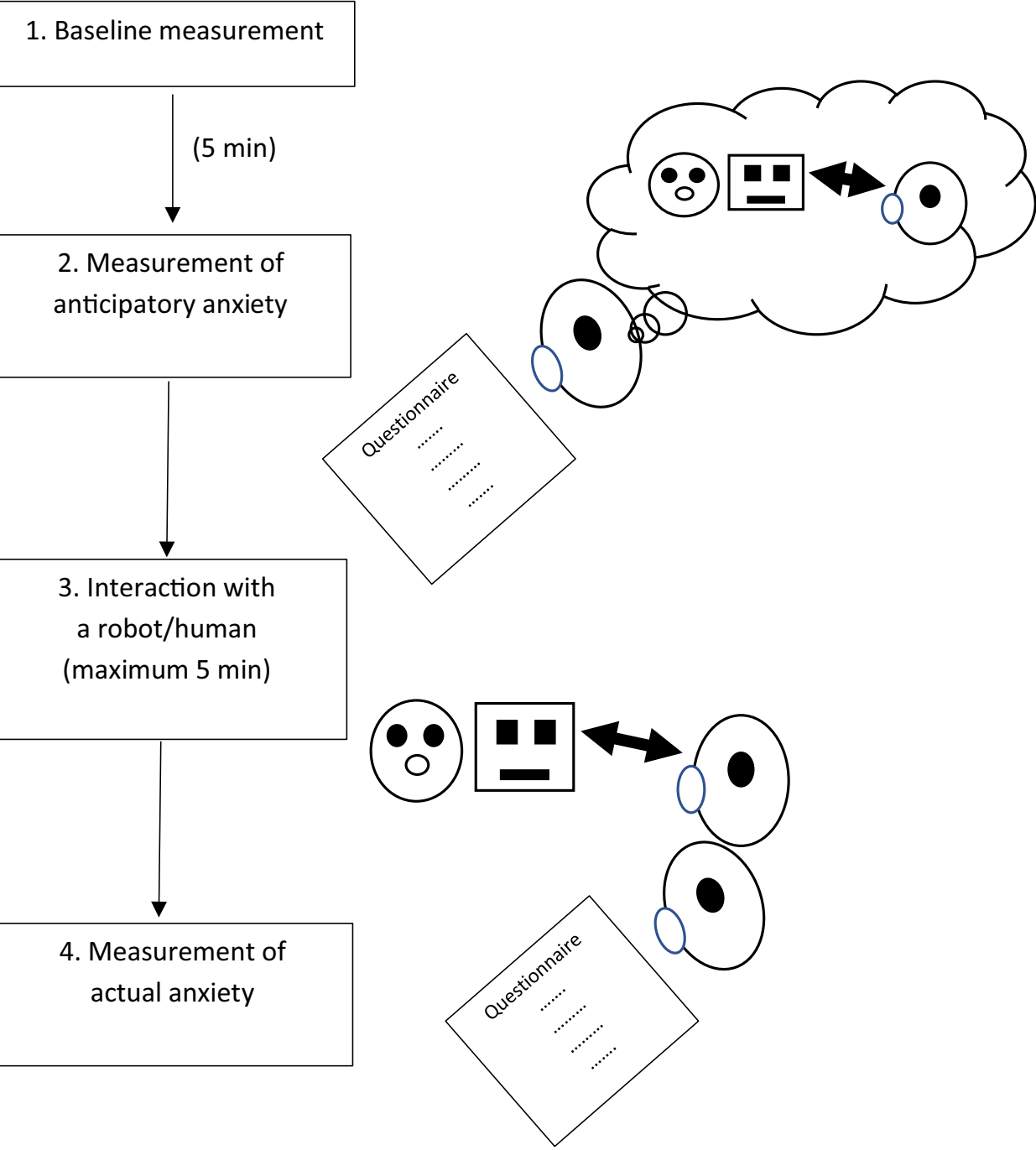


Fig. 3 Overview of the experimental room

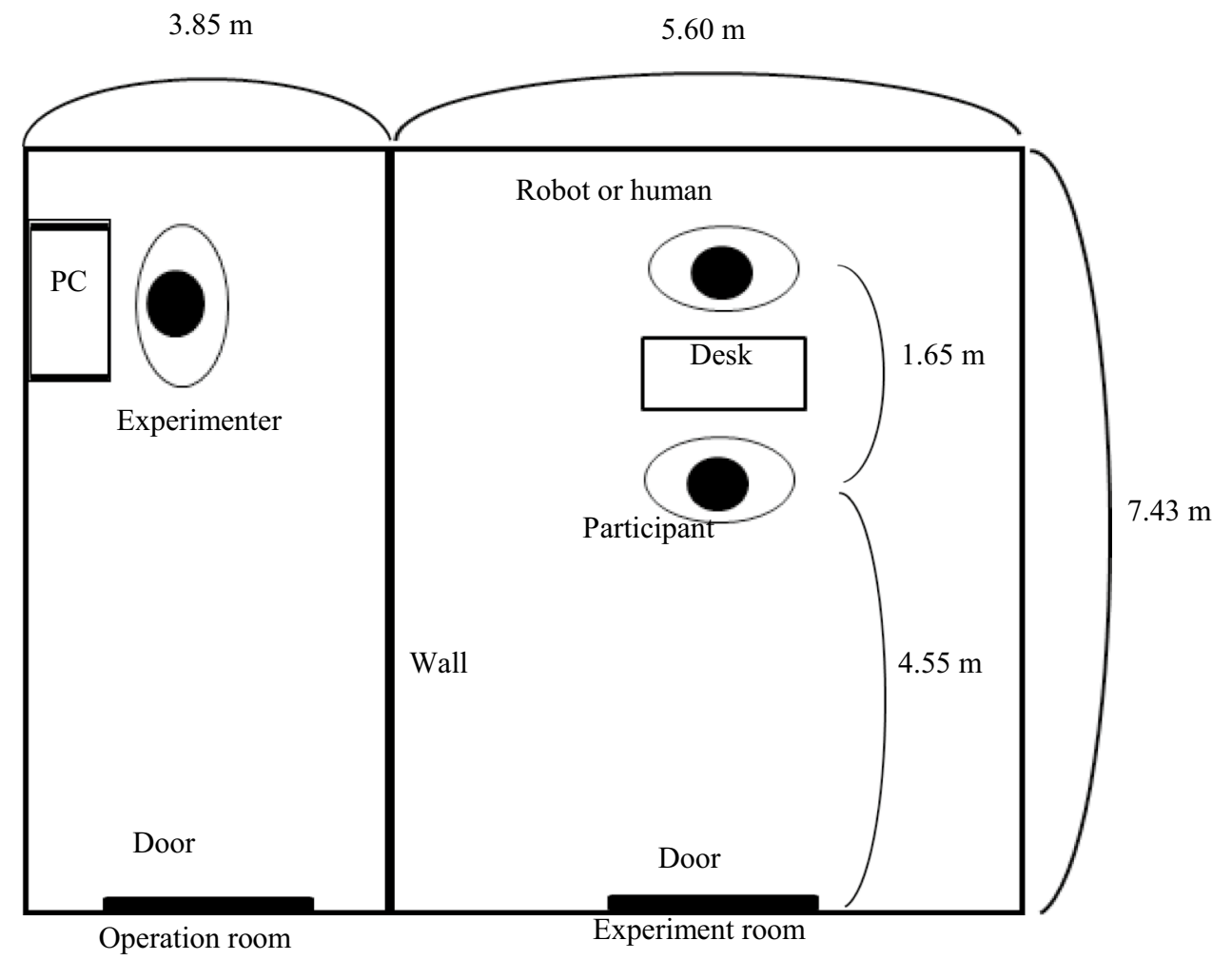

she would enter that room for the purpose of interaction. We also explained that we expected the interaction to continue for $5 \mathrm{~min}$, and that the experimenter would enter the room 5 min later.

When the participant entered the room, he/she started to talk to the counselor. During the discussion, the counselor (a human confederate or a robot) exhibited listening behaviors. That is, the counselor nodded at the end of each paragraph of the participant's utterance. When the participant exhibited a short silence of $2 \mathrm{~s}$, the partner provided a backchannel utterance, "Hai" (in Japanese). Moreover, if the participant stayed silent after the utterance, the counselor encouraged them to talk more by saying "please tell me more about you."

\section{Step 4 Measurement of actual anxiety}

Finally, we measured the participants' anxiety just after the interaction. When the interaction period ended, the experimenter entered the room and escorted the participant out to administer the questionnaire. The participants' tension and state anxiety were measuring using the POMS and STAI-S.

After a 15-min interval, the participant engaged in steps 2-4 under another experimental condition.

The participants' anticipatory anxiety was calculated as the difference in STAI-S and POMS scores between baseline and just after being instructed to interact with the robot and human (and just before interacting); these measures were named "anticipatory anxiety" and "anticipatory tension", respectively. Moreover, the participants' actual state anxiety during interactions was calculated as the difference in STAI-S and POMS scores between baseline and during interactions with the robot and human; these measures were named "actual anxiety" and "actual tension", respectively.

\subsection{Analysis and interpretation}

In addition to statistical tests for the measures above, we adopted effect sizes (e.g., Durlak 2009) for analysis and interpretation. Researchers usually want to show differences between the groups they are studying. Effect sizes provide information about the magnitude and direction of difference between the groups. Even if sample size is small in a study, a sufficiently large effect size can mean the magnitude of difference. On the contrary, even if a study has a large sample size, the small effect size may deny sufficient effect of the groups. The American Psychological Association recommended that researchers should provide effect sizes. Even papers in societies of engineering fields such as IEEE, the world's largest professional association for the advancement of technology, have recently reported effect sizes in their experiments.

Moreover, we used parametric tests for analyses since the basic measures were based on psychological scales, of which scores are regarded as numerical values in the standard of psychology. In this study, we mainly adopted 
analysis of variance as parametric tests based on a psychological convention of 5\% significance levels. As shown in Tables 2 and 3 later, results of this test report the statistical values $(F)$, significant probabilities $(P)$, and partial- $\eta^{2}$ as corresponding effect sizes $\left(\eta_{p}^{2}\right)$.

\section{Hypothesis and predictions}

As mentioned above, people with high social anxiety tend to suffer from anticipatory anxiety. That is, they feel anxious when they expect to communicate with others. We speculated that they would feel less anxious when expecting to communicate with a robot. Based on this idea, we made the following prediction:

Prediction 1: When participants learn that they will be interacting with a robot/human partner, those with higher social anxiety will feel less anticipatory anxiety and anticipatory tension about interacting with a robot compared with a human.

Moreover, we speculated that their level of anxiety would remain the same even after they actually interacted with a partner. That is, those with high social anxiety would feel less anxious after they communicated with a robot compared with a human. Based on this idea, we made the following prediction:
Prediction 2: After interacting with a robot/human partner, participants with higher social anxiety will feel less actual anxiety and actual tension about interacting with a robot compared with a human.

\section{Results}

First, we confirmed that the participants communicated with the robot in the same way as with the human experimenter through video observation. Figure 4 shows the representative scenes of participants communicating with the robot and human experimenter.

\subsection{Scale reliability}

The reliability of each psychological scale used in the experiment was confirmed. Table 1 shows Cronbach's $\alpha$ reliability coefficients for the SADS, POMS, and STAI-S at baseline, just after being instructed to interact (and just before interacting) with the robot, during interaction with the robot, just after being instructed to interact (and just before interacting) with the human, and during interaction with the human. Since the reliability of a scale is guaranteed when the values of the coefficients are higher than .8, the scales showed sufficient internal consistency. In the remainder of the paper, we present the scale scores as the sum of the scores for the corresponding items (the scores of the reverse items are reversed).
Fig. 4 Representative scenes of participants communicating with the robot and human experimenter
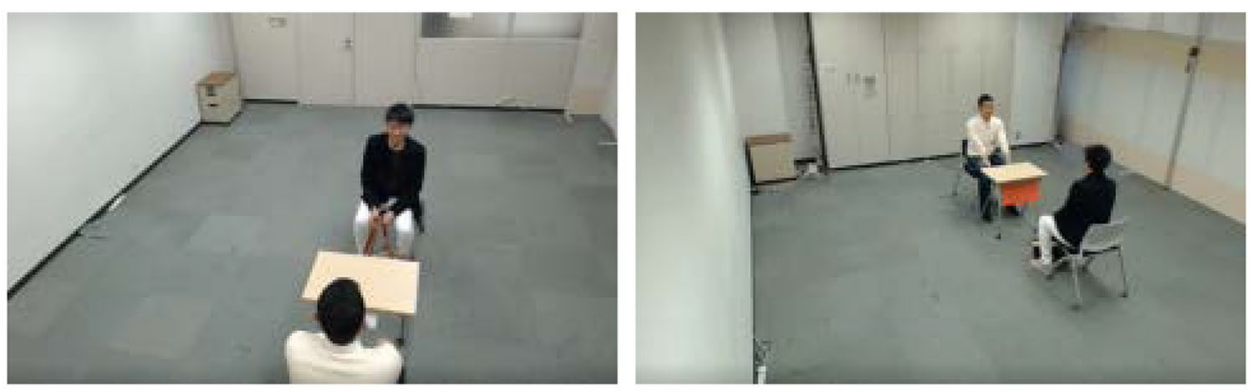

(a) With the human experimenter
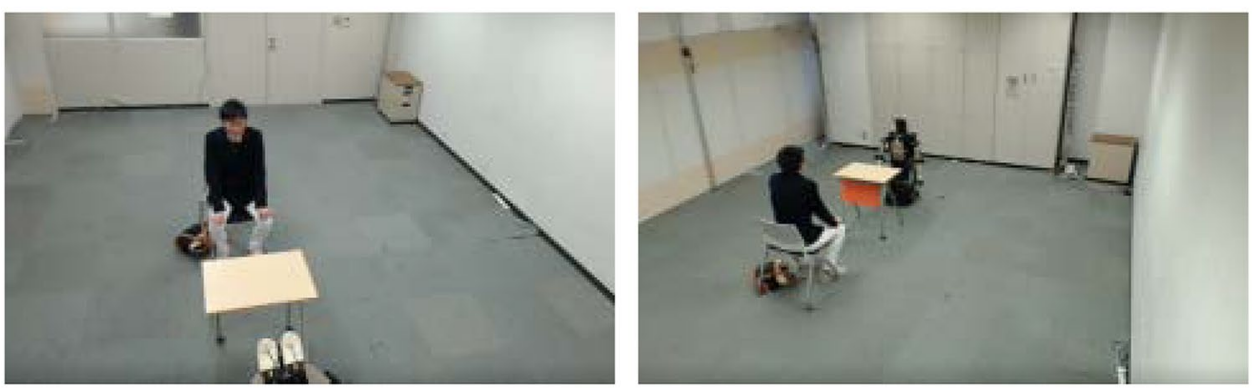

(b) With the robot 
As mentioned in Sect. 2.3, the participant group was divided based on SADS scores as follows

- "lower" social anxiety $(N=12)$ : mean (SD) SADS score was 4.9 (2.7).

- "higher" social anxiety $(N=7)$ : mean $(\mathrm{SD})$ SADS score was 18.1 (5.7).

There was a significant difference on the SADS scores between the two groups $(t=-5.797, p<.001)$.

\subsection{Empirical support for Prediction 1}

For anticipatory anxiety and tension as dependent variables, a mixed analysis of variance (ANOVA) was conducted with lower/higher social anxiety as a between-participant factor and robot/human as a within-participant factor.
Table 2 shows the ANOVA results, and Fig. 5 shows the mean scores and SDs. The interaction effects for both anticipatory anxiety and tension were statistically significant. Regarding the high social anxiety group, simple main effect tests found a statistically significant difference in anticipatory anxiety between the robot/human conditions $(p=.003)$ and a statistically significant trend in anticipatory tension between the robot/human conditions $(p=.063)$. Moreover, a statistically significant trend in anticipatory tension was observed between the low and high social anxiety groups under the robot condition $(p=.051)$.

Therefore, as predicted in Prediction 1, we confirmed that the participants with high social anxiety felt significantly less anticipatory anxiety (we also observed a trend in anticipatory tension) when they anticipated interacting with a robot compared with interacting with a human.
Table 1 Cronbach's $\alpha$ reliability coefficients for the scales used in the present study

\begin{tabular}{|c|c|c|c|c|c|c|c|c|c|c|}
\hline \multirow[t]{2}{*}{ SADS } & \multicolumn{2}{|c|}{ Baseline } & \multicolumn{2}{|c|}{$\begin{array}{l}\text { Just after being } \\
\text { instructed to } \\
\text { interact with the } \\
\text { robot }\end{array}$} & \multicolumn{2}{|c|}{$\begin{array}{l}\text { During interaction } \\
\text { with the robot }\end{array}$} & \multicolumn{2}{|c|}{$\begin{array}{l}\text { Just after being } \\
\text { instructed to } \\
\text { interact with the } \\
\text { human }\end{array}$} & \multicolumn{2}{|c|}{$\begin{array}{l}\text { During interac- } \\
\text { tion with the } \\
\text { human }\end{array}$} \\
\hline & POMS & STAI & POMS & STAI & POMS & STAI & POMS & STAI & POMS & STAI \\
\hline .931 & .942 & .873 & .748 & .879 & .943 & .912 & .955 & .929 & .856 & .888 \\
\hline
\end{tabular}

SADS Social Avoidance and Distress Scale, POMS profile of mood states, STAI state-trait anxiety inventory

\begin{tabular}{|c|c|c|c|c|c|c|}
\hline & \multicolumn{3}{|c|}{ Anticipatory anxiety } & \multicolumn{3}{|c|}{ Anticipatory tension } \\
\hline & $\begin{array}{l}\text { Low/high } \\
\text { social anxiety }\end{array}$ & Robot/human & Interaction & $\begin{array}{l}\text { Low/high } \\
\text { social anxiety }\end{array}$ & Robot/human & Interaction \\
\hline$F$ & .459 & 6.514 & 8.228 & 10.221 & .371 & 6.495 \\
\hline$P$ & .507 & .021 & .011 & .005 & .550 & .021 \\
\hline$\eta_{p}^{2}$ & .026 & .277 & .326 & .375 & .021 & .276 \\
\hline
\end{tabular}

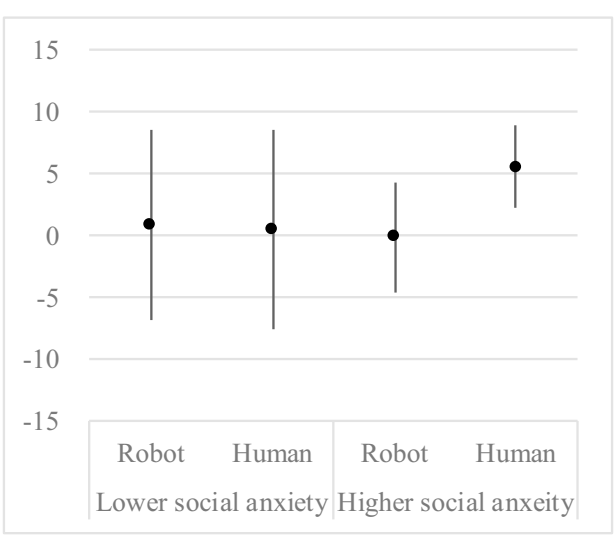

(a) Anticipatory anxiety

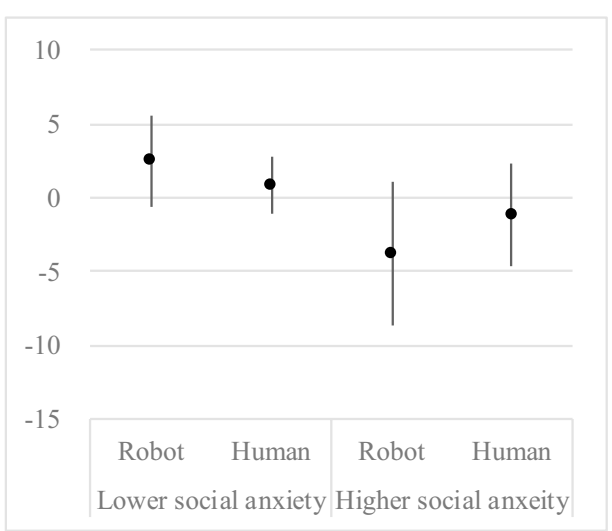

(b) Anticipatory tension
Fig. 5 Means and standard deviations for anticipatory anxiety and tension
Table 2 Results of the analysis of variance for state anxiety and tension in the anticipatory stage 


\subsection{Empirical support for Prediction 2}

For actual anxiety and tension as dependent variables, a mixed ANOVA was conducted with low/high social anxiety as a between-participant factor and robot/human as a within-participant factor.

Table 3 shows the ANOVA results, and Fig. 6 shows the mean scores and SDs. No interaction effects were found in actual anxiety or tension scores in the robot/human interactions. The main effect of robot/human showed a statistically significant trend and a statistically significant difference in actual anxiety and tension, respectively.

Therefore, as predicted in Prediction 2, we found that participants with both high and low social anxiety felt significantly less actual tension (we also observed a trend in actual anxiety) after communicating with a robot compared with a human.

\section{Discussion}

The experiment results showed some statistically significant differences regardless of the small sample size, and sufficiently large effect sizes. Although extreme generalization should be avoided, we can discuss in this stage as follows.

\subsection{Findings}

In the anticipatory stage, the participants in the high social anxiety group felt less anticipatory anxiety and less anticipatory tension toward the robot than the human. They also felt less actual tension toward the robot than the human. These results supported both hypotheses: "Persons with social anxiety would have less 'anticipatory anxiety' when they anticipate meeting with a robot than when they anticipate meeting with a person" and "Persons with social anxiety would have less (actual) anxiety after interacting with a robot than after interacting with a person".

On the other hand, the participants in both the high and lower social anxiety groups reported less anxiety and tension after interacting with the robot than with the human. These results suggest the following:

- Compared with those without, persons with social anxiety prefer to interact with robots over humans when they anticipate interaction.

- Actual interactions with robots can decrease anxiety and tension, regardless of social anxiety.

Moreover, the experiment results suggested that the lower social anxiety group had higher anticipatory and actual tension than the higher social anxiety group. It can be interpreted as follows:
Table 3 Results of the analysis of variance for state anxiety and tension during interactions
Fig. 6 Means and standard deviations for actual anxiety and tension

\begin{tabular}{|c|c|c|c|c|c|c|}
\hline & \multicolumn{3}{|l|}{ Actual anxiety } & \multicolumn{3}{|l|}{ Actual tension } \\
\hline & $\begin{array}{l}\text { Low/high } \\
\text { social anxiety }\end{array}$ & Robot/human & Interaction & $\begin{array}{l}\text { Low/high } \\
\text { social anxiety }\end{array}$ & Robot/human & Interaction \\
\hline$F$ & .229 & 3.588 & .203 & 3.971 & 9.418 & .377 \\
\hline$P$ & .638 & .075 & .658 & .063 & .007 & .547 \\
\hline$\eta_{p}^{2}$ & .013 & .174 & .012 & .189 & .357 & .022 \\
\hline
\end{tabular}

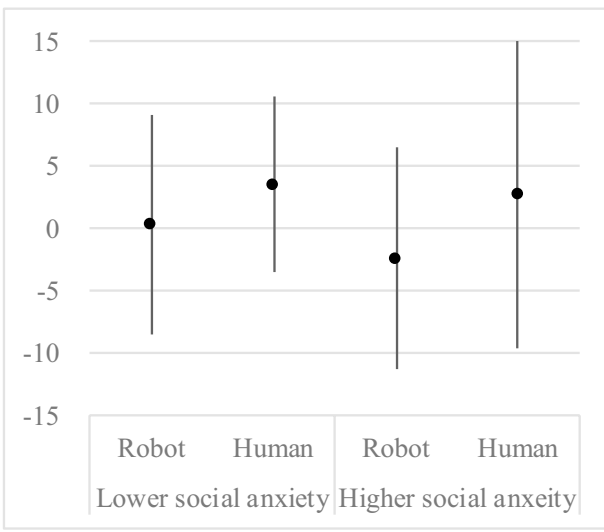

(a) Actual anxiety

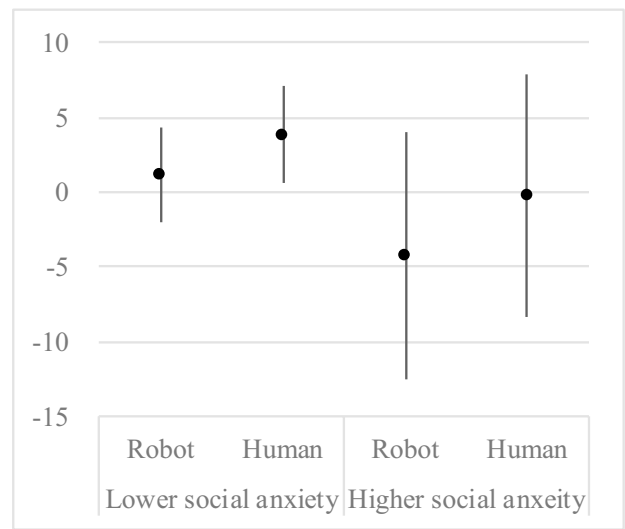

(b) Actual tension 
1. The higher social anxiety group had higher tension than the lower social anxiety group at the stage of the baseline measurement because they felt tension toward the experiment itself. In fact, there was a statistically significant difference on their tensions between these groups $(t=-4.122, p=.001)$.

2. When the higher social anxiety group knew that they faced the robot, their tensions were rapidly decreased. When they knew that they faced a human, their tension were maintained.

3. On the other hand, the lower social anxiety group did not feel tension toward the experiment itself but toward each task in the experiment. It resulted in much difference from the baseline.

4. However, the above tendency was not shown in anticipatory or actual anxiety.

\subsection{Implications}

As mentioned above, persons with social anxiety tend to find social situations difficult because of communication burdens. If robots can mediate between these persons and society, they might be able to encourage such persons to engage in social activities more frequently.

Of course, the present study does not suggest that robots can/should be substituted for human communication partners for persons with social anxiety. Rather, robots can/ should connect such persons with others. For example, communication robots could be introduced into training programs that teach skills to inhibit the symptoms of social anxiety. Although substituting robots for human communication partners is not realistic at the current technological level, robots may be better communication partners than humans in the initial stage of such programs. Substituting humans for robots as such programs progress may enhance and promote skill training.

\subsection{Limitations}

To date, we have only tested within a simple experimental scenario; nevertheless, we believe that the above implications can be sustained for more general situations, similar to those in the present experiment. However, the experiment did have some limitations. First, the sample size was relatively small and limited to Japanese persons. Social anxiety has been shown to be related to culture and ethnicity (Hofmann et al. 2010), so it will be necessary to take these factors into account in future experiments.

Second, the experiment adopted only one type of robot, a human-sized humanoid robot with a mechanical appearance. Therefore, the effect of the size and appearance of robots on humans remains unclear; this will also need to be investigated in future experiments.

\section{Summary and future research}

To assess the influence of social anxiety on human-robot interactions, a psychological experiment was conducted involving a comparison of interactions with a human and a humanoid robot under the assumption that the participant would play the role of a client and the robot or the human would play the role of a counselor. The participants' social anxiety, tension, and state anxiety were measured using corresponding psychological scales (the SADS, POMS, and STAI-S, respectively). The results indicated that, in contrast to persons without, those with social anxiety prefer interacting with robots over humans when anticipating the interaction and that actual interaction with robots can help reduce anxiety and tension in humans, regardless of their level of social anxiety.

Future experiments will be expanded to include several types of robots and participants from other cultures.

Acknowledgments This study was supported by a Grant-in-Aid for Scientific Research (No. 25280095 and 18H04121) from the Japan Society for the Promotion of Science. Moreover, this work was (in part) supported by JST CREST Grant Number JPMJCR17A2, Japan.

\section{Compliance with ethical standards}

Conflict of interest The authors declare that they have no conflict of interest.

Open Access This article is distributed under the terms of the Creative Commons Attribution 4.0 International License (http://creativeco mmons.org/licenses/by/4.0/), which permits unrestricted use, distribution, and reproduction in any medium, provided you give appropriate credit to the original author(s) and the source, provide a link to the Creative Commons license, and indicate if changes were made.

\section{References}

American Psychiatric Association (1994) Diagnostic and statistical manual of mental disorders, 4th edn. American Psychiatric Association, Washington, DC

American Psychiatric Association (2013) Diagnostic and statistical manual of mental disorders, 5th edn. American Psychiatric Association, Washington, DC

Durlak JA (2009) How to select, calculate, and interpret effect sizes. J Pediatr Psychol 34(9):917-928

Hofmann SG, Asnaani A, Hilton DE (2010) Cultural aspects in social anxiety and social anxiety disorder. Depress Anxiety 27:1117-1127

Ishiguro H, Ono T, Imai M, Maeda T, Kanda T, Nakatsu R (2001) Robovie: an interactive humanoid robot. Int $\mathrm{J}$ Ind Robot 28(6):498-503

Kang S-H, Gratch J (2010) Virtual humans elicit socially anxious interactants' verbal self-disclosure. Comput Animat Virtual Worlds $21: 473-482$ 
McNair DM, Lorr M, Droppleman LF (1971) Manual for the profile of mood states. Educational and Industrial Testing Services, San Diego

Nomura T, Kanda T (2015) Influences of evaluation and gaze from a robot and humans' fear of negative evaluation on their preferences of the robot. Int J Social Robot 7(2):155-164

Schneier FR, Johnson J, Hornig CD, Liebowitz MR, Weissman MM (1992) Social phobia: comorbidity and morbidity in an epidemiologic sample. Arch Gen Psychiatry 49(4):282-288

Spielberger CD, Gorsuch RL, Lushene R, Vagg PR, Jacobs GA (1983) Manual for the state-trait anxiety inventory. Consulting Psychologists Press, Palo Alto

Suzuki T, Yamada S, Kanda T, Nomura T (2015) Influence of social avoidance and distress on people's preferences for robots as daily life communication partners. In: Proceedings of the 1st international conference on social robots in therapy and education, New Friends, pp 20-21

Watson D, Friend R (1969) Measurement of social-evaluative anxiety. J Consult Clin Psychol 33:448-457

Publisher's Note Springer Nature remains neutral with regard to jurisdictional claims in published maps and institutional affiliations. 